Chapman University

Chapman University Digital Commons

Physical Therapy Faculty Articles and Research

Physical Therapy

2-2009

\title{
Gastrocnemius-Soleus Muscle Tendon Unit Changes Over the First 12 Weeks of Adjusted Age in Infants Born Preterm
}

Marybeth Grant-Beuttler

Chapman University, beuttler@chapman.edu

Robert J. Palisano

Drexel University

Debra P. Miller

University of Scranton

Barbara Reddien Wagner

University of Scranton

Carolyn B. Heriza

Rocky Mountain University of Health Professions

See next page for additional authors

Follow this and additional works at: http://digitalcommons.chapman.edu/pt_articles

Part of the Musculoskeletal System Commons, Pediatrics Commons, and the Physical Therapy Commons

\section{Recommended Citation}

Grant-Beuttler M, Palisano R, Miller D, Wagner B, Heriza C, Shewokis P. Gastrocnemius/soleus muscle tendon unit changes over the first 12 weeks of adjusted age in infants born preterm. Phys Ther. 2009;89(2): 136-148. doi: 10.2522/ptj.20070306

This Article is brought to you for free and open access by the Physical Therapy at Chapman University Digital Commons. It has been accepted for inclusion in Physical Therapy Faculty Articles and Research by an authorized administrator of Chapman University Digital Commons. For more

information, please contact laughtin@chapman.edu. 


\section{Gastrocnemius-Soleus Muscle Tendon Unit Changes Over the First 12 Weeks of Adjusted Age in Infants Born Preterm}

\section{Comments}

This article was originally published in Physical Therapy, volume 89, issue 2, in 2009. DOI: 10.2522/

ptj.20070306

\section{Creative Commons License}

(c) (i) (9)

This work is licensed under a Creative Commons Attribution-Noncommercial 3.0 License

Copyright

"American Physical Therapy Association"

\section{Authors}

Marybeth Grant-Beuttler, Robert J. Palisano, Debra P. Miller, Barbara Reddien Wagner, Carolyn B. Heriza, and Patricia A. Shewokis 


\section{Research Report}

M Grant-Beuttler, PT, PhD, PCS, is Assistant Professor, Department of Physical Therapy, Chapman University, One University Dr, Orange, CA 92866 (USA). Address all correspondence to $\mathrm{Dr}$ Grant-Beuttler at: beuttler@ chapman.edu.

RJ Palisano, PT, ScD, is Professor, Department of Physical Therapy and Rehabilitation Sciences, Drexel University, Philadelphia, Pennsylvania.

DP Miller, PT, DPT, is Assistant Director of Clinical Education, Department of Physical Therapy, University of Scranton, Scranton, Pennsylvania.

B Reddien Wagner, PT, DPT, MHA, is Director of Clinical Education, Department of Physical Therapy, University of Scranton.

CB Heriza, PT, EdD, FAPTA, is Professor, Doctor of Science Program, Pediatric Therapy, Rocky Mountain University of Health Professions, Provo, Utah.

PA Shewokis, PhD, is Associate Professor, College of Nursing and Health Professions and the School of Biomedical Engineering, Science and Health Systems, Drexel University.

[Grant-Beuttler M, Palisano R], Miller DP, et al. Gastrocnemiussoleus muscle tendon unit changes over the first 12 weeks of adjusted age in infants born preterm. Phys Ther. 2009;89:136-148.]

(C) 2009 American Physical Therapy Association

Post a Rapid Response or find The Bottom Line: www.ptjournal.org

\section{Gastrocnemius-Soleus Muscle Tendon Unit Changes Over the First 12 Weeks of Adjusted Age in Infants Born Preterm}

Marybeth Grant-Beuttler, Robert J Palisano, Debra P Miller, Barbara Reddien Wagner, Carolyn B Heriza, Patricia A Shewokis

Background and Purpose. Differences in the gastrocnemius-soleus muscle and tendon have been documented shortly after birth in infants born preterm compared with infants born at term. Knowledge of muscle tendon unit lengths at term age to 12 weeks of age in infants born preterm may be useful in understanding motor development.

Participants and Method. Gastrocnemius-soleus muscle tendon unit lengths were compared at term age, at 6 weeks of age, and at 12 weeks of age (preterm adjusted age) in 20 infants born full term and 22 infants born preterm.

Results. Significant differences were found between the 2 groups on taut tendon, relaxed muscle length $\left(A_{O}\right)$; taut tendon, stretched muscle length $\left(A_{\text {Max }}\right)$; and muscle stretch ( $A_{O}$ to $A_{\text {Max }}$ ). Infants born preterm demonstrated measures of $A_{O}$ and $A_{\text {Max }}$ in positions of greater plantar flexion compared with infants born full term. Significant differences in measurements of $A_{O}$ were found between term age and 12 weeks of age, indicating that the tendon lengthens during this period for both groups.

Discussion and Conclusion. These results provide knowledge of musculoskeletal development of the gastrocnemius-soleus muscle and tendon. Differences in musculoskeletal measurements are consistent with uterine confinement in the last weeks of full-term gestation. These findings have implications when examining the musculoskeletal system in infants born preterm who are demonstrating functional changes.

For an Invited Commentary and the Author Response, visit www.ptjournal.org. 
$\mathrm{M}$ otor development in infants traditionally has focused on maturation of the central nervous system, and less focus has been placed on posture of the infant and examination of the musculoskeletal system. Posture in infants born preterm, both those at low risk and those at high risk for developmental issues, is generally characterized by more extension $^{1,2}$ and, specifically, by increased trunk extension, ${ }^{3}$ decreased elevation of the hips in a prone position, ${ }^{3-5}$ and lateral (external) rotation of the hips ${ }^{3-5}$ compared $^{-}$ with infants born full term. Prolonged positioning in extension has the potential to alter the musculoskeletal system over the long term. ${ }^{6-8}$ Davis and colleagues ${ }^{9}$ found increased lateral rotation range of motion and increased foot angle during gait in children 3 to 4.5 years of age who had been born preterm and defined as either low risk or high risk. These authors suggested that these changes in range of motion at the hip and in gait are related to a "frog-leg" positioning in the neonatal intensive care unit (NICU). ${ }^{9}$ Heriza $^{10}$ examined supine kicking and reported more dorsiflexion at peak leg flexion in infants born full term compared with infants at low risk born preterm at 40 weeks of gestation. Although the ankle difference was greater than 25 degrees, the statistical power of this analysis was low and differences did not reach significance in that study. The degree of ankle motion during supine kicking found in infants born preterm may potentially be explained by changes in musculoskeletal system development at the ankle.

Posture and positioning observed at birth have been reported to gradually change as infants at low risk and infants at high risk born preterm approach term age ( 40 weeks of gestation). ${ }^{3,4,11}$ Palmer and colleagues ${ }^{2}$ found that differences in arm recoil, arm traction, and leg recoil, which were significantly different at birth between infants born preterm and infants born full term, were no longer significant when the infants born preterm reached term age. By term age, infants born preterm demonstrated increased flexion in their posture. These changes in posture were found to be strongest in infants born preterm with higher birth weights, fewer medical interventions, and later gestational age at birth. ${ }^{2}$

Harris and colleagues ${ }^{11}$ suggested that, over the first year, ankle dorsiflexion decreases a mean of 10 degrees in infants with very low birth weight born before 35 weeks of gestation. Their findings of increased extension at the ankle were consistent with those of Desmond et al ${ }^{12}$ and Grenier, ${ }^{13}$ who suggested that extension increases during the period between birth and term age due to positioning in extension and the force of gravity on the joints. Infants born full term also demonstrated a decrease in dorsiflexion range of motion after birth, perhaps as a consequence of gravity. ${ }^{2,14,15}$ Hoffer $^{14}$ suggested that these changes in range of motion at the ankle in infants born full term were complete by 3 months of age.

Specific changes in the muscle tendon unit (MTU) as a result of positioning could depend on the age of the infants when positioning occurs and not necessarily where these changes occur, in utero or out of utero. Tardieu et $\mathrm{al}^{6}$ and Williams and Goldspink ${ }^{7}$ documented structural changes in the MTU when prolonged shortening or lengthening occurred differently in very young versus adult animals. ${ }^{6,7}$ In very young rabbits $^{6}$ and mice, ${ }^{7}$ tendon lengthening occurred when a muscle was immobilized (18-21 days) in either a lengthened or shortened position, whereas the number of sacromeres decreased in both scenarios. ${ }^{6,7}$ These findings were in contrast to those of adolescent or adult animals that underwent decreased sarcomere number with prolonged immobilization in a shortened position and increased sarcomere number with immobilization in a lengthened position. During the last 4 weeks (28 days) of gestation, the human fetus is confined in a tight uterine space, which decreases the amount of fetal movement and promotes dorsiflexion, putting the gastrocnemiussoleus muscle in a position of prolonged lengthening. ${ }^{16,17}$

Tardieu et al ${ }^{18}$ developed a reliable and valid method of measuring MTU length and muscle belly stretch in the gastrocnemius-soleus muscle that has been applied to infants born preterm and infants born full term. ${ }^{19}$ Their method of measuring MTU length requires 2 goniometric measures. The first measure, $\mathrm{A}_{\mathrm{O}}$, requires palpation of the Achilles tendon while measuring the ankle range with the goniometer. ${ }^{18}$ The measurement is recorded at the first point of maximal Achilles tendon tension while the muscle is relaxed. This measure has been suggested by Tardieu, et $\mathrm{al}^{18}$ to be the point of full tendon lengthening with a relaxed muscle belly. $A_{\text {Max }}$ is a measure of full tendon and muscle belly lengthening. This measurement is taken when the muscle and tendon are moved to maximal stretch, the same way a physical therapist would measure maximum dorsiflexion at the ankle. ${ }^{18}$ Grant-Beuttler et $\mathrm{al}^{19}$ found interrater reliability (intraclass correlation coefficient [ICC $(2,2)]$ ) of .95 to .86 for $A_{\text {Max }}$ and .96 to .97 for $\mathrm{A}_{\mathrm{O}}$ and test-retest reliability (ICC $[3,2])$ of .91 to .97 for $A_{\text {Max }}$ and .97 to .98 for $\mathrm{A}_{\mathrm{O}}$ in infants born preterm and infants born full term.

In previous research using these methods of measuring MTU length, Grant-Beuttler and Shewokis ${ }^{20}$ found that when infants born preterm were examined soon after birth, they demonstrated less maximum dorsiflexion 
compared with infants born full term. Twenty infants at low risk born between 26 and 36 weeks of gestation demonstrated a shorter taut tendon, relaxed muscle length $\left(\mathrm{A}_{\mathrm{O}}\right)$ following birth compared with 21 infants born full term measured within 48 hours of birth. Although differences in both $\mathrm{A}_{\mathrm{O}}$ and $\mathrm{A}_{\mathrm{Max}}$ reached significance, muscle belly stretch $\left(A_{O}\right.$ to $\left.A_{\text {Max }}\right)$ did not reach significance. These results suggest that there is a difference in the gastrocnemius-soleus MTU length between infants born preterm and infants born full term. However, MTU length and muscle belly stretch have not been measured over time to determine whether differences found immediately after birth persist at term age, at 6 weeks of age, or at 12 weeks of age, when the fullterm infant's ankle range of motion stabilizes.

Documenting changes in preterm infants at low risk is especially important secondary to fewer medical issues occurring in these infants and documentation of motor issues occurring at later ages of development. Fewer medical complications and medical interventions increase the likelihood that motor differences are due to preterm birth. In addition, when motor issues are documented, they are found after specific skills have developed in an infant born full term and may not be detected with simple observation. ${ }^{21-25}$ Detecting subtle motor changes is challenging and requires sophisticated systems used in laboratories. ${ }^{21-25}$ Subtle motor issues in infants at low risk are more likely to be missed during a standard physical therapy examination and evaluation, especially early in motor development before skills have developed. Finding a relevant assessment method to detect changes early will increase the likelihood that physical therapy will identify and intervene with subtle issues that may persist.
Examination and evaluation of the gastrocnemius-soleus MTU may be important to detect differences that could alter development of a lowerextremity coordination pattern. During supine kicking, a coordinated lower-extremity movement pattern is learned through repetition. ${ }^{10,25,26}$ This coordinated movement pattern will include increased plantar flexion if the MTU is shortened. As documented by Heriza, ${ }^{10}$ an infant at low risk born preterm may be repeating more plantar flexion during kicking. When the infant is developing a new skill, such as walking, this altered movement pattern may be used. ${ }^{27}$ An altered ankle movement pattern is consistent with the increased frequency of toe-touch foot contact during walking, as documented by Cioni and colleagues. ${ }^{28}$ The relationship between decreased dorsiflexion and persistent toe-walking in infants born preterm also is suggested in the work of Georgieff and associates. ${ }^{29}$ Evaluating the MTU may be useful to physical therapists for predicting which infants born preterm are at risk for altered ankle movements during lower-extremity kicking and active movement during walking.

The purpose of this study was to compare passive gastrocnemius-soleus MTU measurements at the ankle obtained at birth and at 6 weeks and 12 weeks of age between infants born preterm and infants born full term to identify: (1) whether differences in MTU length and muscle belly stretch exist between infants born preterm and infants born full term; (2) whether differences are present in the tendon or the muscle belly, or both; and (3) at what ages changes in the tendon length or the muscle belly, or both, are found. Based on pilot work by Grant-Beuttler and Shewokis, ${ }^{20}$ we hypothesized that differences in the gastrocnemiussoleus MTU would be observed at term age. Whether differences in the MTU between the infants born pre- term and the infants born full term are present at 6 weeks of age and at 12 weeks of age is less clear. Ferrari et $\mathrm{l}^{30}$ presented evidence that by 12 weeks of age, infants at low risk born preterm without neurological impairment demonstrate extremity movements that are similar to movements observed in infants born full term. These observations were based on "general movement assessment," and specific lower-limb kinematics were not measured quantitatively. However, differences in ankle kinematics and the delay in age of walking that have been reported $9,30-32$ suggest that differences in gastrocnemius-soleus MTU length and muscle belly stretch may be present at 12 weeks after term age.

\section{Method}

Participants

Sample size estimates were made prospectively based on a significance criterion of .05 , a power of .80 , and the lowest meaningful effect size (d) of 1.59 for $A_{O}$ and 1.63 for $A_{M a x}$ using G-power (version 2.0) statistical software. ${ }^{33}$ Effect size estimates for measures of muscle length were based on data from the pilot study by Grant-Beuttler and Shewokis. ${ }^{20}$ A sample of 40 infants, with 20 infants born preterm and 20 infants born full term, was required for this study's repeated-measures, longitudinal design.

All infants were recruited to participate while in a hospital setting. Families of infants born full term were recruited from 3 local hospitals. Families of infants born preterm were recruited from NICUs in 2 of the 3 local hospitals. The third local hospital did not have an NICU.

Forty-two infants were included in this study. Twenty infants born following full-term gestation of 38 to 41 weeks were included in a fullterm group. Twenty-two infants born following premature birth at 26 to 36 
weeks of gestation were included in a preterm group. Data collection of infants from preterm births was larger than the original 20 infants secondary to inclusion of infants from multiple births. All infants had normal newborn examinations, with no orthopedic, genetic, or neurological impairments.

Gestational age, birth weight, sex, and method of delivery for both groups of infants are described in Table 1. Mean gestational age for the infants born full term was 39 weeks 3 days $(\mathrm{SD}=6.44$ days, range $=38$ weeks 2 days- 41 weeks 0 days), and mean birth weight was 3,342 $\mathrm{g}$ $(\mathrm{SD}=456.21$, range $=2,750-4,720)$. Eleven of the infants born full term were male, and 9 were female. Five of the infants born full term were born via cesarian section, and 15 were born via normal vaginal delivery. All infants in the full-term group were singleton pregnancies.

Mean gestational age for the infants born preterm was 32 weeks 3 days $(\mathrm{SD}=1$ week 5.43 days, range $=26$ weeks 3 days-34 weeks 5 days), and mean birth weight was $1,854 \mathrm{~g}$ $(\mathrm{SD}=463.14, \quad$ range $=794-2,608)$. Fourteen of the infants born preterm were male, and 8 were female. Fourteen of the infants born preterm were born via cesarian section, and 8 were born by vaginal delivery. Eleven infants born preterm were singleton pregnancies, 3 infants born preterm were a triplet pregnancy, and 8 infants born preterm were twin pregnancies. The infants in the preterm group were eligible to participate in the study if the neonatologist classified them as low risk for a movement disorder. Classification of low risk included limited time on ventilator support, no evidence of orthopedic or genetic impairment, no evidence of neurological complications, and a benign medical course during their stay in the NICU. All infants born preterm were discharged with no indication of continued medical problems.

Infants born full term were recruited within 2 days of birth. Infants born preterm were recruited prior to discharge from the NICU. Posters advertising the project were present in both NICUs and the maternity areas in the 3 hospitals. Nurses in all of the areas were aware of the project and provided with letters for parents that described the project.

Of the 120 families of infants born full term that were contacted by the researchers, 21 families agreed to participate. The family of one infant born full term dropped out prior to data collection. All other infants born full term were measured 3 times, with no attrition in the group.

Of the 33 families of infants born preterm that were contacted by the researchers, 18 families (23 infants, 5 multiple-birth groups) agreed to participate. One family could not be contacted to schedule the first session. The remaining 22 infants in the preterm group were measured 3 times, with no attrition in the group.

\section{Instrumentation}

A 10.16-cm-diameter (4-in-diameter) goniometer* was used to measure ankle range of motion. The goniometer had line designations for each degree. Dorsiflexion measurements were documented as positive angles, and plantar-flexion measurements were documented as negative angles, with a 90-degree angle between the tibia and the lateral foot designated as a neutral position, or 0 degrees.

\section{Procedure}

Informed consent was obtained from a parent prior to data collection, which occurred in the Physical Therapy Movement Laboratory at the Uni-

* Pro-Med Products, 6445 Powers Ferry Rd, \#199, Atlanta, GA 30339. versity of Scranton. Each infant in the full-term group was scheduled for data collection within 7 days after birth, at 6 weeks, and at 12 weeks. Each infant in the pre-term group was scheduled for data collection within 7 days of term age, at 6 weeks adjusted age, and at 12 weeks adjusted age. Families were offered a ride with a professional driving service to and from the laboratory for all 3 visits.

In order to accurately measure gastrocnemius-soleus muscle length and muscle belly stretch, the infant's gastrocnemius-soleus MTU must be relaxed. If the infant was asleep when he or she came to the laboratory, all attempts were made to allow the infant to continue to sleep. If the infant was not asleep, all attempts were made to relax the infant. In an attempt to relax the infant, a parent was asked to cuddle or swaddle him or her in a blanket. In addition, the laboratory was kept warm with a small room heater. All infants were measured when in behavioral state 1 (deep sleep), state 2 (light sleep), or state 4 (quiet, alert) 34,35 because they would most likely have relaxed limbs and less movement when in these states. If any tension was palpated in the limb during measurement, the foot was moved into and out of dorsiflexion and plantar flexion, and measurements were delayed until the infant relaxed. All infant states demonstrated during the measure of muscle length are reported in Table 2 for both groups. Infants were not measured in behavioral state 3 (drowsy), 5 (active, alert), or 6 (crying). ${ }^{34,35}$ If an infant moved into an undesired state, the mother or the researchers assisted the infant in moving into a desired state. No infants were removed from this study because of an inability to get them into or keep them in the desired behavioral state. 


\section{Muscle and Tendon Changes in Infants Born Preterm}

Table 1.

Sex, Gestational Age, Method of Delivery, and Birth Weight for Infants Born Full Term and Infants Born Preterm ${ }^{a}$

\begin{tabular}{|c|c|c|c|c|}
\hline Participant & Sex & Gestational Age (wk) & Method of Delivery & Birth Weight (g) \\
\hline FT-01 & M & $383 / 7$ & NVD & 3,572 \\
\hline FT-02 & M & 40 & NVD & 3,345 \\
\hline FT-03 & $\mathrm{F}$ & 39 & NVD & 2,862 \\
\hline FT-04 & $\mathrm{F}$ & $385 / 7$ & NVD & 3,402 \\
\hline FT-05 & $\mathrm{F}$ & $402 / 7$ & NVD & 3,615 \\
\hline FT-06 & M & $401 / 7$ & NVD & 3,062 \\
\hline FT-07 & M & $392 / 7$ & NVD & 3,827 \\
\hline FT-08 & $\mathrm{F}$ & $395 / 7$ & NVD & 2,750 \\
\hline FT-09 & M & $403 / 7$ & NVD & 4,026 \\
\hline FT-10 & $\mathrm{F}$ & $404 / 7$ & NVD & 4,720 \\
\hline FT-11 & $\mathrm{F}$ & $383 / 7$ & C & 4,267 \\
\hline FT-12 & M & $382 / 7$ & NVD & 3,445 \\
\hline FT-13 & $\mathrm{F}$ & $383 / 7$ & C & 3,260 \\
\hline FT-14 & M & $405 / 7$ & NVD & 3,856 \\
\hline FT-15 & M & $404 / 7$ & NVD & 4,082 \\
\hline FT-16 & M & $386 / 7$ & C & 3,118 \\
\hline FT-17 & M & $383 / 7$ & C & 3,232 \\
\hline FT-18 & $\mathrm{F}$ & $386 / 7$ & C & 3,230 \\
\hline FT-19 & M & $391 / 7$ & NVD & 3,175 \\
\hline FT-20 & $\mathrm{F}$ & 41 & C & 3,827 \\
\hline PT-01 $b$ & $\mathrm{~F}$ & $313 / 7$ & C & 1,191 \\
\hline PT-02 ${ }^{b}$ & $\mathrm{~F}$ & $313 / 7$ & C & 1,446 \\
\hline PT-03 ${ }^{b}$ & $\mathrm{~F}$ & $313 / 7$ & C & 794 \\
\hline PT-04 & $M$ & $324 / 7$ & NVD & 2,410 \\
\hline PT-05 & $\mathrm{F}$ & $321 / 7$ & C & 2,353 \\
\hline PT-06 & $M$ & $304 / 7$ & NVD & 1,474 \\
\hline PT-07c & M & $333 / 7$ & NVD & 2,410 \\
\hline PT-08 ${ }^{c}$ & $M$ & $333 / 7$ & NVD & 2,155 \\
\hline PT-09 & $M$ & $323 / 7$ & C & 1,616 \\
\hline PT-10 & $M$ & $341 / 7$ & NVD & 2,155 \\
\hline PT-11 & $M$ & $263 / 7$ & NVD & 1,049 \\
\hline PT-1 $12^{c}$ & $M$ & $335 / 7$ & C & 2,126 \\
\hline PT-13 ${ }^{c}$ & $\mathrm{M}$ & $335 / 7$ & C & 2,296 \\
\hline PT-14 & M & $341 / 7$ & NVD & 1,899 \\
\hline PT-15 & $M$ & $341 / 7$ & C & 2,041 \\
\hline PT-16 & $M$ & $314 / 7$ & C & 1,588 \\
\hline PT-17 ${ }^{c}$ & $M$ & $326 / 7$ & C & 1,928 \\
\hline PT-1 $18^{c}$ & $M$ & $326 / 7$ & C & 1,673 \\
\hline PT-19 & $\mathrm{F}$ & $324 / 7$ & C & 2,070 \\
\hline PT-20 & $\mathrm{F}$ & 31 & C & 1,219 \\
\hline PT-21 & $\mathrm{F}$ & $345 / 7$ & NVD & 2,608 \\
\hline PT-22 & $\mathrm{F}$ & $334 / 7$ & C & 2,296 \\
\hline
\end{tabular}

${ }^{a} \mathrm{FT}=$ full term, $\mathrm{PT}=$ preterm, $\mathrm{M}=$ male, $\mathrm{F}=$ female, $\mathrm{NVD}=$ normal vaginal delivery, $\mathrm{C}=$ cesarean section.

${ }^{b}$ Triplet birth.

${ }^{c}$ Twin birth. 
Table 2.

Behavioral States Exhibited by Infants Born Full Term $(n=20)$ and Infants Born Preterm $(n=22)$ During Measurement of Muscle Length at Each Age Measured ${ }^{a}$

\begin{tabular}{|c|c|c|c|c|c|c|}
\hline \multirow[b]{2}{*}{ Behavioral States } & \multicolumn{2}{|c|}{ Term Age } & \multicolumn{2}{|c|}{6 wk of Age } & \multicolumn{2}{|c|}{12 wk of Age } \\
\hline & $\begin{array}{l}\text { Full-term } \\
\text { Group }\end{array}$ & $\begin{array}{c}\text { Preterm } \\
\text { Group }\end{array}$ & $\begin{array}{l}\text { Full-term } \\
\text { Group }\end{array}$ & $\begin{array}{l}\text { Preterm } \\
\text { Group }\end{array}$ & $\begin{array}{l}\text { Full-term } \\
\text { Group }\end{array}$ & $\begin{array}{c}\text { Preterm } \\
\text { Group }\end{array}$ \\
\hline 1 & $1(5 \%)$ & & & & & \\
\hline 2 & $2(10 \%)$ & $3(13.5 \%)$ & & $2(9 \%)$ & $1(5 \%)$ & \\
\hline 4 & & $1(4.5 \%)$ & $8(40 \%)$ & $10(46 \%)$ & $15(75 \%)$ & $16(73 \%)$ \\
\hline 1,2 & $12(60 \%)$ & $12(55 \%)$ & $3(15 \%)$ & $2(9 \%)$ & & \\
\hline $1-3$ & $1(5 \%)$ & & & $1(4.5 \%)$ & & \\
\hline $1,2,4$ & & & $1(5 \%)$ & & & \\
\hline 2,3 & $2(10 \%)$ & $3(13.5 \%)$ & & $1(4.5 \%)$ & & \\
\hline 2,4 & & $1(4.5 \%)$ & $1(5 \%)$ & & & \\
\hline $2-4^{b}$ & & $1(4.5 \%)$ & $1(5 \%)$ & $4(18 \%)$ & & \\
\hline $2-5$ & & & $1(5 \%)$ & & & \\
\hline 3,4 & & $1(4.5 \%)$ & $3(15 \%)$ & $1(4.5 \%)$ & & $1(4.5 \%)$ \\
\hline $3-5^{c}$ & $1(5 \%)$ & & & & & $2(9 \%)$ \\
\hline 4,5 & $1(5 \%)$ & & $2(10 \%)$ & $1(4.5 \%)$ & $4(20 \%)$ & $3(13.5 \%)$ \\
\hline
\end{tabular}

a Number of infants in each group of behavioral states listed, with percentage of infants in parentheses.

${ }^{b}$ Primarily in behavioral states 2 and 4 .

c Primarily in behavioral state 4 .

As shown in Table 2, infants in the full-term group exhibited more sleep states (states 1 and 2) during the term measurement period and more alert states (states 4 and 5) by the 12-week measurement period. The infants in the preterm group demonstrated similar trends toward sleep states in the term measurement period, and most infants demonstrated alert states in the 12-week measurement period. Infants born preterm demonstrated an ability to regulate their states in a manner similar to that of the infants born full term. This ability of the infants born preterm to maintain and regulate their states is another indication of their physiological stability. No infants in either group demonstrated state 6 (crying), and no infant spent more than brief periods in state 5 (active, alert). Documentation of the states by both groups of infants suggests that measurement could be completed when the infant was in a relaxed state at all ages.
Each infant's ankle was passively moved through the available range of motion before taking measurements in an attempt to ensure the infant was relaxed. Ankle range of motion was measured as described by Norkin and White. ${ }^{36}$ Figures 1 and
2 illustrate measurement of MTU length. The first measurement, $\mathrm{A}_{\mathrm{O}}$, is the angle between the foot and the leg when the foot is moved from plantar flexion to dorsiflexion and the palpation of the Achilles tendon reveals the first point of maximum

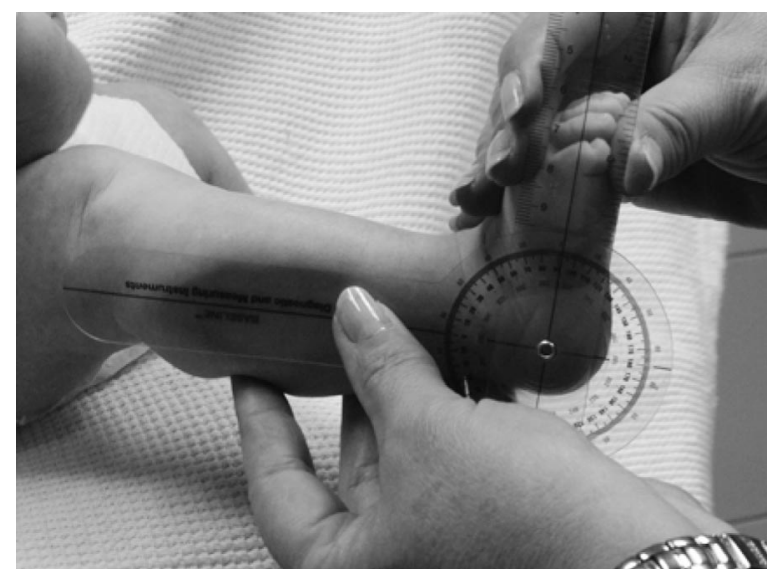

Figure 1.

Measurement of $A_{O}$ muscle length. The $A_{O}$ goniometric measurement is recorded when the Achilles tendon becomes taut. This measure represents slack removed from the tendon, with a relaxed muscle belly. 


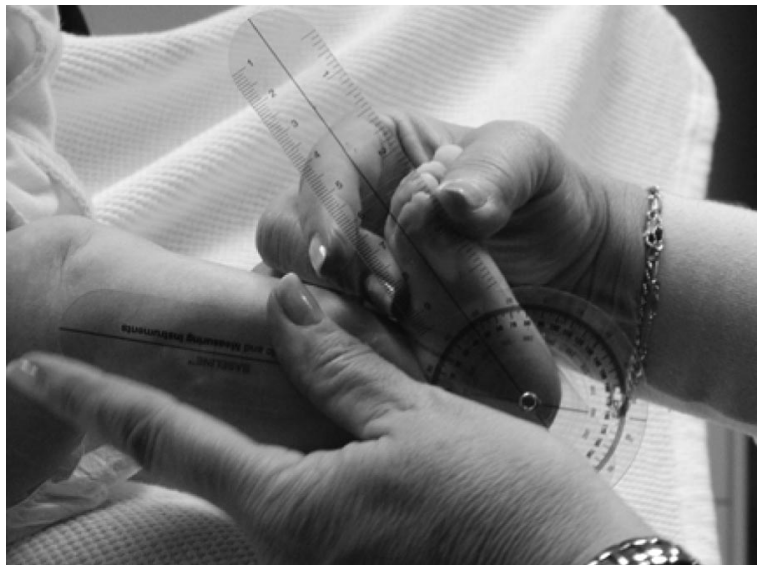

Figure 2.

Measurement of $A_{\text {Max }}$ muscle length. The $A_{\text {Max }}$ goniometric measurement is recorded when the muscle belly and the tendon are stretched to their maximum. This measure represents slack removed from the tendon and full stretch of the muscle belly.

tautness (Fig. 1). This measurement reflects the point in ankle range where slack is removed from the Achilles tendon and the muscle belly of the gastrocnemius-soleus muscle is relaxed. A second measurement, $A_{\text {Max }}$, then was taken after the foot was gently moved into maximum dorsiflexion (Fig. 2). When the foot was moved into maximum dorsiflexion, the infant's behavior was closely monitored to ensure no discomfort resulted from this movement. This measurement reflected a fully lengthened Achilles tendon and maximal stretch in the muscle belly of the gastrocnemius-soleus muscle. The knee was positioned into maximum extension during measurement of both $\mathrm{A}_{\mathrm{O}}$ and $\mathrm{A}_{\text {Max }}$.

Knee extension limitations can be present in newborn infants who are developmentally normal, and have been documented as a mean of -15.3 degrees $^{37}$ to -21.4 degrees. ${ }^{15}$ Brown and Swenson ${ }^{38}$ examined dorsiflexion in newborns with knee extension and knee flexion to 90 degrees. In girls, dorsiflexion was 60 degrees with the knee extended and 63 degrees with the knee flexed. The difference between dorsiflexion with and without knee flexion was
57 and 59 degrees, respectively, in boys. Based on the data from Brown and Swenson ${ }^{38}$ and knowing knee extension limitation is less than 45 degrees, we suggest the change in the MTU length measures with the knee extended as far as possible would be a difference of no more than half of that found with 90 degrees of knee flexion, ${ }^{38}$ or 1 to 1.5 degrees. The accepted standard for measuring ankle range of motion in the newborn is to extend the knee as far as possible. ${ }^{11,14,15,37}$ Applying this clinical standard to this study allowed greater ease in transferring applications into clinical practice.

Each measurement was taken twice by 2 examiners who were blinded to the other examiner's measurements. For each measurement, the infant's foot was placed in a plantar-flexed position, moved toward dorsiflexion for the measurement of $\mathrm{A}_{\mathrm{O}}$, and then moved into maximum dorsiflexion for the measurement of $A_{M a x}$. The foot was returned to a plantar-flexed position, and then the second measurements were taken in the same manner. Only one examiner measured at a time, and the examiners did not watch each other measure. Each examiner recorded 2 measures of $\mathrm{A}_{\mathrm{O}}$ and $\mathrm{A}_{\mathrm{Max}}$ on a sticky note that was added to the research folder for each infant after measurements were complete. If the examiner recorded 2 measurements for either $A_{O}$ or $A_{\text {Max }}$ that were more than 4 degrees different, a third measurement was taken, and the examiner recorded the 2 measurements that were most similar. A third measurement was obtained during $15 \%$ of the measurements for both examiners on either $\mathrm{A}_{\mathrm{O}}$ or $\mathrm{A}_{\text {Max }}$. Once the measurements were complete, each infant was dressed, and the next session was scheduled. Blinding of infant gestational age group was not possible because each examiner was able to visually distinguish among infants in each group based on experience, particularly at term age. The ICC $(2,1)$ for reliability of $A_{O}$ between the 2 raters on 100 of the 126 measurements was .99 (95\% confidence interval $=.98-.99)$. The ICC $(2,1)$ for reliability of $\mathrm{A}_{\mathrm{Max}}$ between the 2 raters on 99 of the 126 measurements was $.95(95 \%$ confidence interval $=$ $.93-.97)$.

\section{Data Analysis}

Data for measurements of $\mathrm{A}_{O}$ and $A_{\text {Max }}$ were entered into SPSS version 12.0. ${ }^{\dagger}$ The 2 measurements of $A_{O}$ and $A_{\text {Max }}$ were averaged after they were entered into SPSS. If a third measurement was taken, the 2 most similar measurements were averaged. A third variable, muscle belly stretch ( $A_{O}$ to $\left.A_{M a x}\right)$, was created by subtracting the measurement of $A_{O}$ from the measurement of $A_{M a x}$. This variable represents muscle extensibility, the distance the muscle belly fibers stretched. All 3 values were entered for each infant at each age measured, resulting in 126 measurements for each measure of MTU length.

${ }^{\dagger}$ SPSS Inc, 233 S Wacker Dr, Chicago, IL 60606 . 
Differences between infants born preterm and infants born full term for each of the 3 measures $\left(A_{O}, A_{\text {Max }}\right.$, and $A_{O}$ to $\left.A_{M a x}\right)$ were analyzed using a $2 \times 3$ (term $\times$ age), mixed-model analysis of variance (ANOVA). In this statistical model, the first factor (term) was a between-subjects factor that was a random effect, and the second factor (age) was a withinsubjects factor that was a fixed effect. Because 3 separate ANOVAs were performed to analyze these data, a Bonferroni adjustment was made to the alpha level $(.05 / 3=$ .0167). Follow-up analysis for main effects of age was performed using the Tukey honestly significant difference test. Effect size for the main effects and interactions in the ANOVA were reported using partial eta ${ }^{2}$. Partial eta ${ }^{2}$ represents the amount of the measure and error variance that can be attributed to term, age, or the interaction of these main effects. Cohen d effect sizes and 95\% confidence interval for these effect sizes were determined for the differences in means between the full-term infants and the preterm infants using the Effect Size Calculator. ${ }^{39}$

\section{Role of Funding Source}

This study was funded by the Edward J. Leahy Center for Faculty Research Awards through the University of Scranton. The funding from this grant provided transportation to and from the laboratory setting for all 3 visits, if agreed to by the family. An approved car seat was provided for the families to use when using the provided transportation. Following completion of all 3 data collection meetings, each family was given a gift card for a local store that sold various newborn items. This funding source in no way biased the outcome of this investigation.

\section{Results}

The means and standard deviations for the 3 measures at term age, 6 weeks of age, and 12 weeks of age

Table 3.

Mean Values and Standard Deviations (in Degrees) of Muscle Tendon Unit Measures at Term Age, 6 Weeks of Age, and 12 Weeks of Age ${ }^{a}$

\begin{tabular}{|c|c|c|c|}
\hline \multirow[b]{2}{*}{ Measure } & $\begin{array}{l}\text { Full-term Group } \\
(\mathbf{n}=\mathbf{2 0})\end{array}$ & $\begin{array}{l}\text { Preterm Group } \\
(\mathbf{n}=\mathbf{2 2})\end{array}$ & \multirow{2}{*}{$\begin{array}{c}\text { Cohen d } \\
\text { (95\% Confidence } \\
\text { Interval) }\end{array}$} \\
\hline & $\bar{X}(S D)$ & $\bar{X}(S D)$ & \\
\hline \multicolumn{4}{|l|}{$A_{\circ}$} \\
\hline Term & $-3.7(3.1)$ & $-17.0(3.8)$ & $3.82(2.74-4.75)$ \\
\hline $6 \mathrm{wk}$ & $-3.3(2.0)$ & $-14.8(4.2)$ & $3.44(2.43-4.32)$ \\
\hline $12 \mathrm{wk}$ & $-2.5(0.8)$ & $-14.1(2.3)$ & $6.61(4.97-8.00)$ \\
\hline \multicolumn{4}{|l|}{$A_{\operatorname{Max}}$} \\
\hline Term & $48.0(3.8)$ & $39.3(6.0)$ & $1.71(0.98-2.39)$ \\
\hline $6 \mathrm{wk}$ & $46.5(3.2)$ & $41.1(5.7)$ & $1.15(0.48-1.78)$ \\
\hline $12 \mathrm{wk}$ & $47.4(3.3)$ & $43.0(4.7)$ & $1.07(0.41-1.70)$ \\
\hline \multicolumn{4}{|l|}{$A_{O}$ to $A_{\operatorname{Max}}$} \\
\hline Term & $51.7(4.9)$ & $56.3(7.4)$ & $0.73(0.09-1.34)$ \\
\hline $6 \mathrm{wk}$ & $49.8(3.6)$ & $55.9(5.2)$ & $1.35(0.66-2.00)$ \\
\hline $12 \mathrm{wk}$ & $49.9(3.3)$ & $57.1(5.6)$ & $1.55(0.83-2.21)$ \\
\hline
\end{tabular}

${ }^{a}$ Plantar flexion is denoted by negative degrees, and dorsiflexion is denoted by positive degrees. $A_{O}=$ taut tendon, relaxed muscle belly; $A_{M a x}=$ taut tendon, stretched muscle belly; $A_{O}$ to $A_{M a x}=$ muscle belly stretch.

are presented in Table 3. Cohen $\mathrm{d}$ effect sizes ranged from 0.73 to 6.61 (Tab. 3), suggesting differences in the means between the groups were medium-large to very large. ${ }^{40}$ Measurements of $\mathrm{A}_{\mathrm{O}}, \mathrm{A}_{\mathrm{Max}}$, and $\mathrm{A}_{\mathrm{O}}$ to $A_{\text {Max }}$ for both groups over all 3 ages are represented in Figures 3 and 4. Differences in MTU length $\left(A_{O}\right.$ and $\left.A_{\text {Max }}\right)$ and muscle belly stretch $\left(A_{O}\right.$ to $\left.A_{\text {Max }}\right)$ between infants born preterm and infants born full term were all significant (Tab. 4). At term age, 6 weeks of age, and 12 weeks of age, infants born preterm demonstrated values of $A_{O}$ and $A_{\text {Max }}$ in positions of greater plantar flexion, with a larger range of $A_{O}$ to $A_{M a x}$, compared with values in infants born full term (Tab. 3). Partial eta ${ }^{2}$ ranges were as high as .90 for $A_{O}$, suggesting that full term versus preterm explained $90 \%$ of the variability observed in $A_{O}$ (Tab. 4).

A significant difference in the main effect of age was found for $A_{O}$ (Tab. 4). As infants aged, the measure of $A_{O}$ was in a position of more dorsiflexion, representing an increase in tendon length. A Tukey honestly significant difference post boc analysis revealed a significant difference in $\mathrm{A}_{O}$ only between term age and 12 weeks of age.

\section{Discussion}

The results support the hypothesis that, at term age, infants born preterm and infants born full term differ in gastrocnemius-soleus MTU length and muscle belly stretch and that these differences persist at 6 weeks and 12 weeks of age. Infants born preterm demonstrate less MTU length with a taut tendon, relaxed muscle belly $\left(\mathrm{A}_{\mathrm{O}}\right)$, and a taut tendon, stretched muscle belly $\left(\mathrm{A}_{\mathrm{Max}}\right)$ at term age and at 12 weeks of age. The increased range of muscle belly stretch ( $A_{O}$ to $A_{M a x}$ ) indicates that the muscle belly stretched more in the infants born preterm from term age to 12 weeks of age. Assuming more muscle belly stretch is primarily the result of a longer muscle belly with more sarcomeres or longer sarcomere length, ${ }^{6,7,18,41}$ the tendon may be shorter in the infant born preterm. 


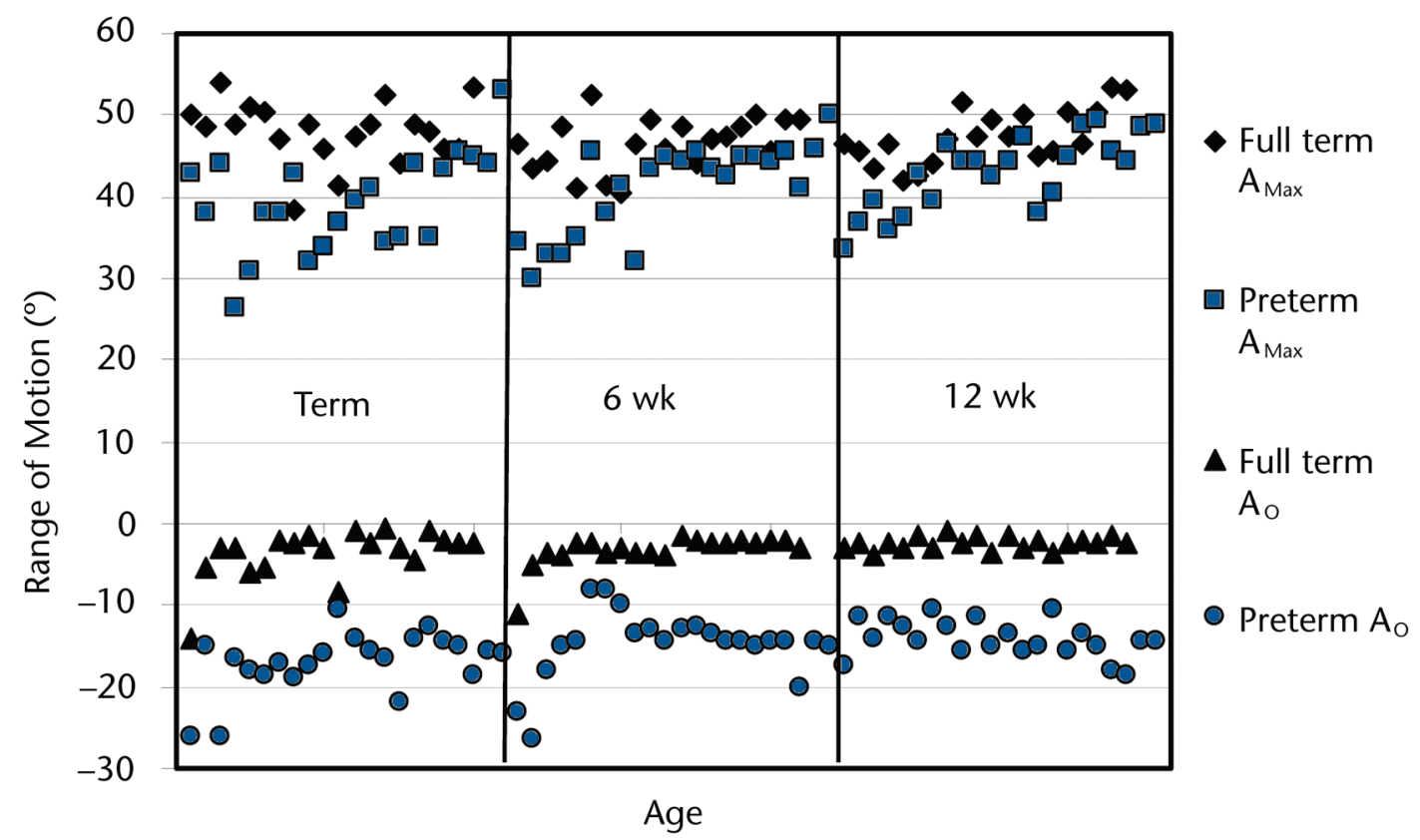

Figure 3.

Measurements of taut tendon, relaxed muscle belly $\left(A_{O}\right)$ and taut tendon, stretched muscle belly $\left(A_{\text {Max }}\right)$ at term age, 6 weeks of age, and 12 weeks of age in infants born full term and infants born preterm. Plantar flexion is represented by negative degrees, and dorsiflexion is represented by positive degrees.

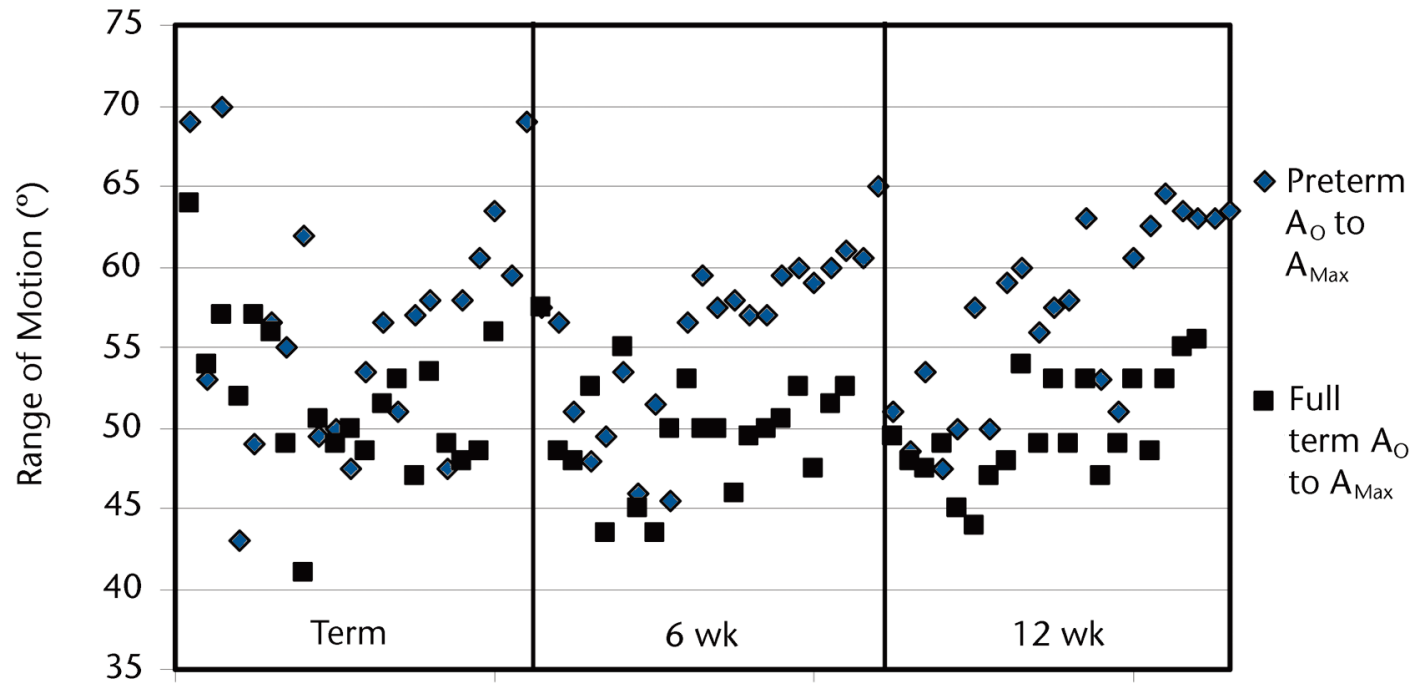

Age

Figure 4.

Measurements of muscle belly stretch (taut tendon, relaxed muscle belly $\left[\mathrm{A}_{\mathrm{O}}\right]$ to taut tendon, stretched muscle belly $\left[\mathrm{A}_{\mathrm{Max}}\right]$ ) at term age, 6 weeks of age, and 12 weeks of age in infants born full term and infants born preterm. 
Table 4.

Main Effects for Term, Age, and Interaction on Analyses of Variance for 3 Measures of the Muscle Tendon Unit ${ }^{a}$

\begin{tabular}{|c|c|c|c|}
\hline Measure & $\begin{array}{c}\text { Between } \\
\text { Terms }\end{array}$ & Between Ages & Interaction \\
\hline \multirow[t]{4}{*}{$A_{\circ}$} & $F_{1,40}=358.27$ & $F_{2,39}=6.23$ & $F_{2,39}=1.33$ \\
\hline & $P<.001$ & $P=.005$ & $P=.28$ \\
\hline & Power $=1.00$ & Power $=.87$ & Power $=.27$ \\
\hline & Partial eta ${ }^{2}=.90$ & Partial eta ${ }^{2}=.24$ & Partial eta ${ }^{2}=.064$ \\
\hline \multirow[t]{4}{*}{$A_{\operatorname{Max}}$} & $F_{1,40}=30.65$ & $F_{2,39}=2.92$ & $F_{2,39}=3.65$ \\
\hline & $P<.001$ & $P=.066$ & $P=.035$ \\
\hline & Power $=1.00$ & Power $=.54$ & Power $=.56$ \\
\hline & Partial eta ${ }^{2}=.43$ & Partial eta ${ }^{2}=.13$ & Partial eta ${ }^{2}=.14$ \\
\hline \multirow[t]{4}{*}{$\mathrm{A}_{\mathrm{O}}$ to $\mathrm{A}_{\mathrm{Max}}$} & $F_{1,40}=25.20$ & $F_{2,39}=.89$ & $F_{2,39}=.72$ \\
\hline & $P<.001$ & $P=.42$ & $P=.49$ \\
\hline & Power $=1.00$ & Power $=.19$ & Power $=.16$ \\
\hline & Partial eta ${ }^{2}=.39$ & Partial eta ${ }^{2}=.044$ & Partial eta ${ }^{2}=.036$ \\
\hline
\end{tabular}

${ }^{a} \mathrm{~A}_{\mathrm{O}}=$ taut tendon, relaxed muscle belly; $\mathrm{A}_{\mathrm{Max}}=$ taut tendon, stretched muscle belly; $\mathrm{A}_{\mathrm{O}}$ to $A_{\text {Max }}=$ muscle belly stretch. Power was determined retrospectively.

Our results suggest that only one measure $\left(\mathrm{A}_{\mathrm{O}}\right)$ demonstrated a change over age. Lengthening of the tendon occurred in all infants, both preterm and full term, between term age and 12 weeks of age. This change could be explained by numerous factors, including decreased knee flexion contraction from term age to 12 weeks of age, tendon growth, and an MTU adaptation to more kicking. Increased tendon length and decreased knee flexion contraction are not convincing explanations because $\mathrm{A}_{\mathrm{Max}}$ also would have changed from term age to 12 weeks of age. ${ }^{42}$ An increase in range of dorsiflexion movements between 6 and 9 weeks of age has been reported by Ferrari et $\mathrm{al}^{30}$ and is consistent with the increase in tendon length we found in passive measures. The increase in tendon length could be an adaptive response to inertial forces on the ankle during kicking between term age and 12 weeks of age.

Group $\times$ age interactions were not statistically significant at a level of .0167 for any of the 3 measures of the MTU. However, the interaction for $A_{\text {Max }}$ was .035, which would have been accepted as significant if we had not adjusted our alpha level. These measures are found to move in opposite directions, decreasing in the full-term group and increasing in the preterm group, when the means are examined (Tab. 3), suggesting that these measures could be moving in different patterns. The patterns of changes in MTU over the 3 ages should be interpreted cautiously due to a possible type II error. Power is reported in Table 4 and should be examined when interpreting the results of the statistical analyses and applying the results.

The results from this study are consistent with the findings from our previous study. ${ }^{20}$ As shown in Table 5 and Figure 5, our results for $A_{O}$, $A_{\text {Max }}$, and $A_{O}$ to $A_{\text {Max }}$ are very similar and support the reliability of our data using the procedure developed by Tardieu and colleagues. ${ }^{18}$ An unexpected finding was the similarity between the preterm infants at birth in our previous study ${ }^{20}$ and the preterm infants at term age in the current study. Although some authors ${ }^{2,4}$ have reported postural changes from birth to term age in infants born preterm, we found no evidence of a similar change in the gastrocnemiussoleus MTU. Our findings at the ankle do not correspond with either the assumption that gastrocnemiussoleus MTU changes from birth to term age as the result of gravity causing more ankle extension ${ }^{12,13}$ or the assumption that gastrocnemiussoleus MTU changes from birth to term age as the result of increased flexor tone causing more ankle flexion. $^{2-4}$ The significant difference in muscle belly stretch $\left(A_{O}\right.$ to $\left.A_{M a x}\right)$ between the 2 groups in this study differs from the findings of our previous study. ${ }^{20}$ Post boc statistical power for the previous study's comparison of $A_{O}$ to $A_{\text {Max }}$ between full-term infants and preterm infants was low (.06), suggesting the possibility of a type II error. In this study, we had a similar number of infants, but measurements were repeated 3 times, resulting in higher statistical power.

If a goal of therapy is to re-create the MTU length observed in infants born full term while intervening with the infant born preterm, the age window for successful therapeutic implementation may be limited. The changes we found in this study between the infants born full term, who underwent a prolonged stretch in utero during the last 4 to 6 weeks of gestation, and the infants born preterm, who did not undergo this prolonged stretch, is the same response Tardieu et $\mathrm{al}^{6}$ and Williams and Goldspink ${ }^{7}$ found in the animal studies. Our data suggest that the adaptation of muscle in the full-term group results in lengthening of the tendon and shortening of the muscle belly in comparison with the infants born preterm. Because the response in these infants is similar to the animal model for very young animals, there may be a period where the adaptation to stretch will change and an intervention will no longer lead to the same 


\section{Muscle and Tendon Changes in Infants Born Preterm}

Table 5.

Means, Standard Deviations, Medians, Interquartile Ranges, and 95\% Confidence Intervals (in Degrees) for 3 Measures of the Muscle Tendon Unit in Our Pilot Study 20 and the Current Study ${ }^{a}$

\begin{tabular}{|c|c|c|c|c|}
\hline \multirow[b]{2}{*}{ Measure } & \multicolumn{2}{|c|}{ Full term } & \multicolumn{2}{|c|}{ Preterm } \\
\hline & Pilot Study & Current Study & Pilot Study & Current Study \\
\hline \multicolumn{5}{|l|}{$A_{O}$} \\
\hline $\bar{x}$ & -6.04 & -3.70 & -18.05 & -17.00 \\
\hline SD & 6.92 & 3.12 & 8.17 & 3.77 \\
\hline Median & -4.00 & -2.75 & -16.50 & -16.25 \\
\hline Interquartile range & 3.50 & 3.25 & 7.00 & 3.63 \\
\hline $95 \%$ confidence interval & -9.20 to -2.90 & -5.16 to -2.24 & -21.87 to -14.23 & -18.67 to -15.33 \\
\hline \multicolumn{5}{|l|}{$A_{\text {Max }}$} \\
\hline $\bar{x}$ & 49.19 & 48.03 & 37.85 & 39.30 \\
\hline SD & 4.59 & 3.78 & 8.76 & 6.00 \\
\hline Median & 48.00 & 48.75 & 40.00 & 38.75 \\
\hline Interquartile range & 7.50 & 4.38 & 7.75 & 9.13 \\
\hline $95 \%$ confidence interval & 47.10 to 51.28 & 46.26 to 49.79 & 33.75 to 41.95 & 36.63 to 41.96 \\
\hline \multicolumn{5}{|l|}{$A_{O}$ to $A_{\operatorname{Max}}$} \\
\hline $\bar{x}$ & 55.24 & 51.73 & 55.90 & 56.30 \\
\hline SD & 7.16 & 4.88 & 7.85 & 7.39 \\
\hline Median & 54.00 & 51.00 & 55.00 & 56.50 \\
\hline Interquartile range & 10.50 & 6.88 & 6.50 & 11.00 \\
\hline $95 \%$ confidence interval & 51.98 to 58.50 & 49.44 to 54.01 & 52.22 to 59.58 & 53.02 to 59.57 \\
\hline
\end{tabular}

${ }^{a} A_{O}=$ taut tendon, relaxed muscle belly; $A_{\operatorname{Max}}=$ taut tendon, stretched muscle belly; $A_{O}$ to $A_{M a x}=$ muscle belly stretch.

adaptive change found in the very young infant, but will switch to the adaptation found in adult animals and humans. Different responses to immobilization at different ages suggest that intervention to mimic the lengthening in infants born preterm should occur as soon as possible if it is to be similar to the lengthening that occurs in utero in infants born full term.

Muscle tendon unit length measures at the ankle should be used to document gastrocnemius-soleus muscle changes in infants born preterm from birth to term age and beyond. Successful dorsiflexion positioning using a splint that limits plantar flexion and allows active dorsiflexion in the NICU or shortly after discharge should result in MTU lengths closer to those found in the full-term group. ${ }^{43,44}$ The preterm infants in this study and in our previous study ${ }^{20} \mathrm{did}$ not receive physical therapy positioning programs in the NICU, so positioning in neutral and dorsiflexed ankle positions by a physical therapist needs to be explored. This study does support the idea that MTU length and muscle belly stretch can be used as reliable clinical measures to document preterm MTU changes in the NICU and during early intervention. In addition, to increase reliability when performing measurements of ankle range of motion, therapists should repeat each measurement twice to compare consistency, monitor behavioral state during measurements, and move the ankle through the full available range of motion prior to taking the measurements to decrease creep.

The gastrocnemius-soleus MTU length in infants born preterm may poten- tially affect lower-extremity coordinated movement patterns and motor learning. A shorter gastrocnemiussoleus MTU would lead to movement in more plantar flexion during lower-extremity kicking. Use of a lower-extremity kicking pattern with increased ankle plantar flexion could explain, at least partially, ankle plantar flexion during supine kicking, ${ }^{10}$ new walkers' initial foot contact in plantar flexion (toe touch), ${ }^{28}$ and persistent toe walking in preterm infants with decreased passive dorsiflexion. ${ }^{29}$ This method of measuring gastrocnemius-soleus MTU length may be a quick method available to physical therapists to predict which infants are at continued risk for changes in learning coordinated movement patterns such as kicking, which ultimately could alter the development of motor skills, such as walking, at later ages. Research is 


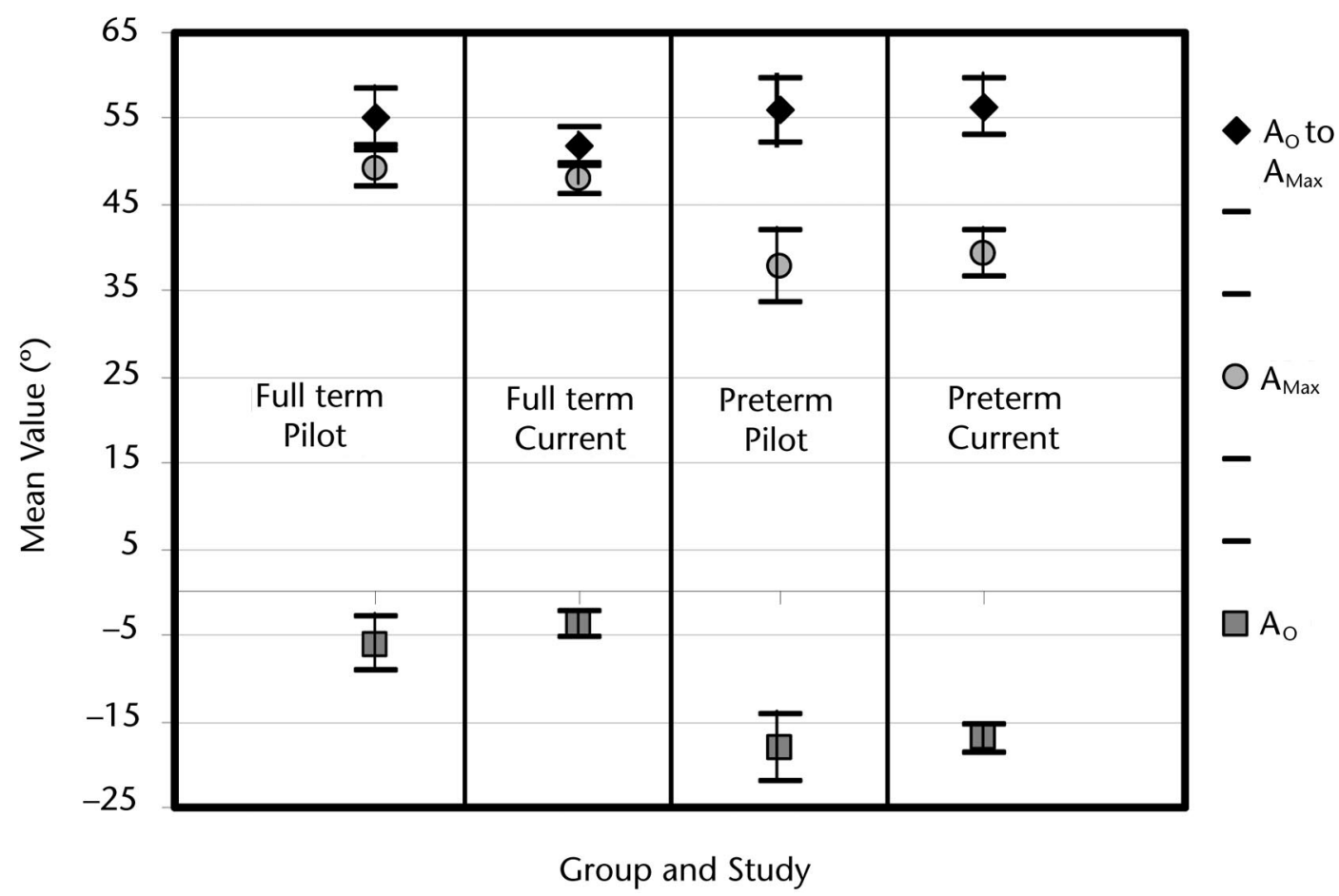

Figure 5 .

Mean values and $95 \%$ confidence intervals for measures of taut tendon, relaxed muscle belly $\left(A_{O}\right)$; taut tendon, stretched muscle belly $\left(A_{\text {Max }}\right)$; muscle belly stretch $\left(A_{O}\right.$ to $A_{\text {Max }}$ ) from pilot study 20 and current study.

needed to investigate changes in MTU length at later ages and the effects of these changes on development of supine kicking, standing, and walking function.

There are limitations in the design of this study. In addition to the lack of full knee extension and the inability to blind the examiners to the groups described earlier, another possible limitation of the results of this study is the low statistical power for the main effect of age. Our a priori power was based on the effect sizes found in our initial study ${ }^{20}$ with respect to differences between infants born preterm and infants born full term and not on changes over age. To adequately power a follow-up study, a minimum of 40 subjects would be needed in each of the groups of infants, or larger age intervals could be compared to increase the likelihood of detecting a difference over age. The lack of signifi- cance found in this study over age needs to be interpreted with caution, considering the possibility of a type II error.

Another limitation of this study is the lack of understanding of MTU length measures past the age of 12 weeks or in infants born preterm who are not low risk. It is possible that the MTU issues resolve or continue at older ages and similar changes are found in infants who are at higher risk for developmental issues. In addition, comparison of the same infants born preterm from birth to term age would be more convincing evidence that MTU lengths from birth until term age did not change during this period.

\section{Conclusions}

Muscle tendon unit lengths in the gastrocnemius-soleus muscle are different between infants born full term and infants born preterm. In- fants born preterm demonstrate shorter tendon length and shorter overall tendon length and muscle belly stretch, but more muscle belly stretch. These differences are present at term age and remain until 12 weeks of age (adjusted for the preterm group). Measurements of MTU length at term age in this study are similar to previous measurements obtained in preterm infants at birth. These measures can be used reliably to document changes in the MTU during an infant's stay in the NICU and during early intervention followup post-discharge. Additional research is needed to document the effects of the MTU lengths on active movement at these ages and beyond.

Dr Grant-Beuttler and Dr Palisano provided concept/idea/research design. Dr GrantBeuttler, Dr Palisano, Dr Heriza, and Dr Shewokis provided writing. Dr GrantBeuttler, Dr Miller, and Dr Reddien Wagner provided data collection, project manage- 
ment, and participants. All authors provided data analysis. Dr Grant-Beuttler provided fund procurement and facilities/equipment.

This project was approved by institutional review boards at the University of Scranton, Scranton-Temple Residency Program, and Drexel University. It also received approval from the Community Medical Center's Executive Board.

This research was presented at the International Conference for Society for Chaos Theory in Psychology \& Life Sciences; July 2729, 2007; Orange, California, and as a poster presentation at the Combined Sections Meeting of the American Physical Therapy Association; February 1-5, 2006; San Diego, California.

This study was funded by an Edward R Leahy Jr Center Research Award.

This article was received October 9, 2007, and was accepted November 8, 2008.

DOI: 10.2522/ptj.20070306

\section{References}

1 Carter RE, Campbell SK. Early neuromuscular development of the premature infant. Phys Ther. 1975;55:1332-1341.

2 Palmer PG, Duowitz LM, Verghote M, Dubowitz V. Neurological and neurobehavioral differences between preterm infants at term and full-term newborn infants. Neuropediatrics. 1982;13:183-189.

3 Vaivre-Douret L, Ennouri K, Jrad I, et al. Effects of positioning on the incidence of abnormalities of muscle tone in low-risk, preterm infants. Eur J Paediatr Neurol. 2004;8:21-34.

4 Lacey JL, Henderson-Smart DJ, Edwards DA. A longitudinal study of early leg postures of preterm infants. Dev Med Child Neurol. 1990;32:151-163.

5 Downs JA, Edwards AD, McCormick DC, et al. Effect of intervention on development of hip posture in very preterm babies. Arch Dis Child. 1991;66:797-801.

6 Tardieu C, Tabary JC, Tabary C, Heut de la Tour E. Comparison of the sarcomere number and adaptation in young and adult animals: influence of tendon adaptation. J Physiol (Paris). 1977;73:1045-1055.

7 Williams PE, Goldspink G. Changes in sarcomere length and physiological properties in immobilized muscle. J Anat. 1978; 127:459-468.

8 Tabary JC, Tabary C, Tardieu C, et al. Physiological and structural changes in the cat's soleus muscle due to immobilization at different lengths by plaster casts. J Physiol. 1972;224:231-244.

9 Davis DW, Thelen E, Keck J. Treadmill stepping in infants born prematurely. Early Hum Dev. 1994;39:211-223.
10 Heriza C. Comparisons of leg movements in preterm infants at term with healthy full-term infants. Phys Ther. 1988;68: 1687-1693.

11 Harris MB, Simons CJR, Ritchie SK, et al. Joint range of motion development in premature infants. Ped Phys Ther. 1990;9: 185-191.

12 Desmond MM, Wilson GS, Alt EJ, Fisher ES. The very low birth weight infant after discharge from intensive care: anticipatory health care and developmental course. Curr Probl Pediatr. 1980;10:1-59.

13 Grenier A. Prevention of early hip deformities in the brain-damaged newborn infant: Little's disease without scissoring? Ann Pediatr (Paris). 1988;35:423-427.

14 Hoffer MM. Joint limitations in newborns. Clin Orthop Relat Res. 1980;148:94-96.

15 Broughton NS, Wright J, Menelaus MB. Range of knee motion in normal neonates. J Pediatr Orthop. 1993;13:263-264.

16 D'Elia A, Pighetti M, Moccia G, Santangelo N. Spontaneous motor activity in normal fetuses. Early Hum Dev. 2001;65:139-147.

17 Roodenburg PJ, Wladimiroff JW, van Es A, Prechtl HFR. Classification and quantitative aspects of fetal movements during the second half of normal pregnancy. Early Hum Dev. 1991;25:19-35.

18 Tardieu C, Heut de la Tour, Bret MD, Tardieu G. Muscle hypoextensibility in children with cerebral palsy, I: clinical and experimental observations. Arch Phys Med Rebabil. 1982;63:97-102.

19 Grant-Beuttler M, Leininger PM, Palisano RJ. Reliability of a measure of muscle extensibility in full-term and preterm newborns. Phys Occup Ther Pediatr. 2004;24: 173-185.

20 Grant-Beuttler M, Shewokis PA. Muscle tendon unit comparisons between infants born preterm and infants born full term: a pilot study. Ped Phys Ther. 2007;19: 309-314.

21 Fallang B, Saugstad O, Gregaard J, HaddersAlgra M. Kinematic quality of reaching movements in preterm infants. Pediatr Res. 2003;54:429-435.

22 Van der Fits I, Flikweert E, Stremmelaar E, et al. Development of postural adjustments during reaching in preterm infants. Pediatr Res. 1999;46:1-7.

23 Hadders-Algra M, Brogren E, Katz-Salamon M, Forssberg H. Periventricular leucomalacia and preterm birth have different detrimental effects on postural adjustments. Brain. 1999;22:727-740.

24 Sagnol C, Debillon T, Debû B. Assessment of motor control using kinematic analysis in preschool children born very preterm. Dev Psychobiol. 2007; 49:421-432.

25 Geerdink J, Hopkins B, Beek W, Heriza C. The organization of leg movements in preterm and full-term infants after term age. Dev Psychobiol. 1996;29:335-351.

26 Droit S, Boldrini A, Cioni G. Rhythmic leg movements in low-risk and brain-damaged preterm infants. Early Hum Dev. 1996; 44:201-213.
27 Thelen E, Bradshaw G, Ward J. Spontaneous kicking in month-old infants: manifestation of a human central locomotor pattern. Behav Neural Biol. 1981;32:45-53.

28 Cioni G, Duchini F, Milianti B, et al. Differences and variations in the patterns of early independent walking. Early Hum Dev. 1993;35:193-205.

29 Georgieff M, Bernbaum J, HoffmanWilliamson M, Daft A. Abnormal truncal muscle tone as a useful early marker for developmental delay in low birth weight infants. Pediatrics. 1986;77:659-663.

30 Ferrari F, Cioni G, Einspieler C, et al. Cramped synchronized general movements in preterm infants as an early marker for cerebral palsy. Arch Pediatr Adolesc Med. 2002;156:422-423.

31 Jeng SF, Yau KI, Liao HF, et al. Prognostic factors for walking attainment in very lowbirthweight preterm infants. Early Hum Dev. 2000;59:159-173.

32 Lacey J. Very low-birthweight preterm infants walk later than term infants, but most are walking by 18 months. Aust J Physiother. 2001;47:65.

33 Buchner A, Faul F, Erdfelder E. G-Power: A Priori, Post Hoc, and Compromise Power Analysis for Windows-based Operating System. Trier, Germany: University of Trier; 1996.

34 Brazelton TB. Neonatal Behavioral Assessment Scale. 2nd ed. Philadelphia, PA: JB Lippincott Co; 1984. Clinics in Developmental Medicine, No. 88.

35 Als H. Neonatal Individualized Developmental Care and Assessment Program (NIDCAP). Boston, MA: Children's Hospital; 1984.

36 Norkin CC, White JD. Measurement of Joint Motion: A Guide to Goniometry. 3rd ed. Philadelphia, PA: FA Davis Co; 2003.

37 Waugh KG, Minkel JL, Parker R, Coon VA. Measurement of selected hip, knee, and ankle joint motions in newborns. Phys Ther. 1983;63:1616-1621.

38 Brown GA, Swenson DR. A descriptive system for lower extremity evaluation in children: data for the newborn infant. Ortbopedics. 2000;23:111-115.

39 Effect Size Calculator. Available at: http:// davidmlane.com/hyperstat/effect-size.html. Accessed March 27, 2003.

40 Cohen J. Statistical Power Analysis for the Behavior Sciences. 2nd ed. Hillsdale, NJ: Erlbaum; 1988.

41 Brand PW, Beach RB, Thompson DE. Relative tension and potential excursion of muscles in the forearm and hand. J Hand Surg. 1981;6:209-219.

42 Huijing PAJBM, Rozendal RH, Heslinga JW, Willems MET. Skeletal muscle reaction to growth and immobilization. J Rehabil Res Dev. 1994;43:30-31.

43 Noonan K, Richards B. Nonsurgical management of idiopathic clubfoot. J Am Acad Ortbop Surg. 2003;11:392-402.

44 Bensahel H, Guillaume A, Czukonyi Z, Desgrippes Y. Results of physical therapy for idiopathic clubfoot: a long-term follow-up study. J Pediatr Orthop. 1990;10:189-192. 